\title{
Stereotactic radiotherapy on brain metastases with recent hemorrhagic signal: STEREO-HBM, a two-step phase 2 trial
}

Paul Lesueur ${ }^{1,2}$, William Kao ${ }^{1}$, Alexandra Leconte ${ }^{3}$, Julien Geffrelot ${ }^{1}$, Justine Lequesne ${ }^{3}$, Joëlle Lacroix ${ }^{4}$, Pierre-Emmanuel Brachet ${ }^{3,5}$, loana Hrab ${ }^{5}$, Philippe Royer ${ }^{6}$, Bénédicte Clarisse ${ }^{3}$ and Dinu Stefan ${ }^{1,7^{*}}$

\begin{abstract}
Background: Brain metastases often occur in cancer evolution. They are not only responsible for death but also for disorders affecting the quality of life and the cognitive functions.

Management of brain metastases usually consists in multi-modality treatments, including neurosurgery, whole brain radiotherapy (WBRT), and more recently radiosurgery (SRS) or fractionated stereotactic radiotherapy (FSRT), systemic treatment (chemotherapy or targeted therapy), combined or not with corticosteroids. Almost 20\% of brain metastases can present recent (within 15 days) bleeding signs on neuro-imagery. In these conditions, WBRT is the usual treatment. Yet, patients may benefit from a more aggressive strategy with SRT or FSRT. However, these options were suspected to possibly major the risk of brain haemorrhage, although no scientifically proven. Radiation oncologists therefore usually remain reluctant to deliver SRS/FSRT for bleeding brain metastases. It is therefore challenging to establish a standard of care for the treatment of bleeding brain metastases. We propose a phase II trial to simultaneously assess safety and efficacy of FSRT to manage brain metastases with hemorrhagic signal.

Methods: The STEREO-HBM study is a multicenter two-step non-randomised phase II trial addressing patients with at least one bleeding brain metastasis out of a maximum of 3 brain metastases. Each brain metastasis will be treated with $30 \mathrm{~Gy}$ in 3 fractions for 1 week.

The main endpoint is based on both safety and efficacy endpoints as proposed by Bryant and Day's design. Safety endpoint is defined as the rate of bleeding complications 4 months post-FSRT while efficacy endpoint is defined as the 6-month local control rate. Multi-modal MRI will be used to assess intra-tumoral hemorrhagic events before and after treatment. Patients' quality of life will also be assessed.
\end{abstract}

Discussion: Management of bleeding brain metastases is still debated and poorly explored in clinical trials. There is sparse and weak data on the signification of pretreatment intra-tumour haemorrhagic signs or on the risk of brain bleeding complications after FSRT.

We expect this first prospective phase 2 trial in this particular setting will allow to clarify the place of FSRT to optimally manage bleeding brain metastases.

Trial registration: NCT 03696680, registered October, 4, 2018.

Protocol version: Version 2.1 dated from 2018/11/09.

Keywords: Stereotactic radiotherapy, Brain metastases, Bleeding, Quality of life

\footnotetext{
* Correspondence: d.stefan@baclesse.unicancer.fr

${ }^{1}$ Radiation Oncology Department, Centre François Baclesse, F-14000 Caen,

France

${ }^{7}$ Radiation Oncology Department, Centre François Baclesse, 3 Avenue du

Général Harris, F-14076 Caen Cedex 05, France

Full list of author information is available at the end of the article
}

(c) The Author(s). 2020 Open Access This article is distributed under the terms of the Creative Commons Attribution 4.0 International License (http://creativecommons.org/licenses/by/4.0/), which permits unrestricted use, distribution, and reproduction in any medium, provided you give appropriate credit to the original author(s) and the source, provide a link to the Creative Commons license, and indicate if changes were made. The Creative Commons Public Domain Dedication waiver (http://creativecommons.org/publicdomain/zero/1.0/) applies to the data made available in this article, unless otherwise stated. 


\section{Background}

Brain metastases occur in $20-40 \%$ of cancer patients. They represent the most common manifestation of intracranial malignancy [1]. They are an important cause of mortality and morbidity. Indeed, brain metastases can result in devastating clinical consequences, such as sensitive-motor defect, cognitive disturbance, social relationship deterioration. Without any specific treatment, patients with brain metastases usually survive for 1 to 2 months $[2,3]$. For these patients with brain evolution of their cancer, death results from the extra-cerebral disease progression in most of cases, but from complications related to brain lesions progression in at least 25$50 \%$ of cases $[4,5]$.

Brain metastases exhibit highly variable revelations modes. They can be asymptomatic or otherwise occur more abruptly. An epileptic seizure or loss of consciousness may reveal brain damage. In that latter case, it is estimated that 1.9 to $10 \%$ of these symptoms are associated with intra-tumoral haemorrhage [6]. Bleeding risk varies depending on histology. For example, melanoma metastases are macroscopically bleeding in $35.7 \%$ of cases, whereas 2.9 and $4.7 \%$ of metastases from adenocarcinoma or anaplastic carcinoma are bleeding, respectively [7]. Overall, almost $20 \%$ of brain metastases can present recent (within 15 days) bleeding signs on neuroimaging (Magnetic Resonance Imaging (MRI) or Scan).

Although radiosurgery (SRS) or fractionated stereotactic radiotherapy (FSRT) is now the mainstay of treatment for brain oligo-metastases (3-5 metastases), allowing a 12-month local control greater than 75\% [8], whole brain radiotherapy (WBRT) still remains the usual treatment of haemorrhagic brain metastases, despite its poor efficacy, namely a 6-month and 12-month local control rate of 37 and 15\%, respectively [9]. This attitude is consistent with the report of the French High Authority of Health (HAS) which does not support radiosurgery for the treatment of haemorrhagic brain metastases (HAS report 2001). It is based on the results from a retrospective study (131 metastases on 54 patients) [10]: haemorrhage was identified in $7.4 \%$ of the metastases before radiosurgery and in $18.5 \%$ of the metastases after radiosurgery. Since this publication, although it did not clearly demonstrate a relationship between radiosurgery and the risk of haemorrhage, FSRT/SRS is suspected to increase the risk of brain haemorrhage. Furthermore, in spite of several reports of intra-tumor haemorrhage after radiosurgery of brain metastases, radiosurgery was not shown to increase the incidence of haemorrhage. Thus, among melanoma patients carrying brain metastases [11], the rate of intra-tumor haemorrhage was shown to be similar before and after treatment by stereotactic Gammaknife (23.7\% vs. $15.2 \%, p=0.89)$; the presence of intra-tumoral bleeding before treatment was not found to major the risk of bleeding after treatment $(p=0.9)$. According to some authors, the occurrence of posttreatment bleeding would not be related to the achievement of radiosurgery, but rather to the intrinsic sensitivity of the tumor to bleed [12].

Besides these conflicting findings, it has to be highlighted that most of these studies were conducted exclusively with SRS (a single fraction issued) and from either a Gammaknife ${ }^{\bullet}$ or a linear adapted accelerator. To date, there are no specific available data for FSRT (several fractions) with Cyberknife ${ }^{\circledR}$, a newer technology.

Overall, radiation oncologists generally remain reluctant to deliver FSRT on hemorrhagic brain metastases. Therefore, the standard treatment remains panencephalic irradiation, even if it is clearly not optimal.

In this context, there is a real need to establish a standard management of hemorrhagic brain metastases, notably using more innovative radiotherapy techniques like FSRT.

In order to specifically document the interest of FSRT in the management of hemorrhagic brain metastases, we propose the first non-randomized phase 2 prospective trial aiming to simultaneously evaluate safety and efficacy of this treatment. In addition, it will accurately document, using multi-modal MRI, intra-tumoral hemorrhagic events before and after treatment. Patients' quality of life before and after treatment will be also assessed.

\section{Methods/design \\ Trial objectives \\ Primary objective}

The main objective is based on joint primary endpoints of safety and efficacy of FSRT for patients with bleeding brain metastases at diagnosis, as proposed by the Bryant-and-Day design [13].

The safety endpoint is the rate of hemorrhagic complications (MRI signal modifications with or without clinical manifestation) occurring within 4 months after the end of FSRT $[14,15]$, defined as the proportion of patients with at least one target brain metastasis with a bleeding complication within 4 months post-FSRT.

The efficacy endpoint is the local control rate of irradiated target lesions (all irradiated brain lesions with stable size or size increase less than 25\%) 6 months after the end of FSRT, using RECIST 1.1 criteria.

Targets lesions correspond to all irradiated lesion regardless the presence of a bleeding signal.

\section{Secondary objectives}

The secondary objectives are to evaluate:

- safety profile (all acute and late toxicities according to EORTC criteria) 
- intra-cerebral progression-free survival (excluding irradiated lesions)

- extra-cerebral progression-free survival

- overall survival

- quality of life evolution at short, mid and long term using EORTC QLQ-C30 and QLQ-BN20 questionnaires

- survival without any toxicity (grade $\geq 2$ ) including quality of life (QoL) impairment (of $\geq 10$ points out of a 100 -point scale in at least one dimension of QoL), nor tumor progression (Q-TWIST)

- the prevalence of modifications after FSRT on morphological, functional and spectro-MRI parameters

\section{Study population}

Eligibility criteria are detailed in Table 1 . More specifically, the targeted patients had to carry up to 3 brain metastases of solid tumor [16, 17], measuring $5-30 \mathrm{~mm}$ in diameter, eligible to stereotactic radiotherapy, of which at least one lesion presented signs of intra-tumor bleeding [18] before stereotactic irradiation.

\section{Trial design}

The study protocol and this manuscript have been written in accordance with standard protocol items, namely recommendations for interventional trials (SPIRIT).

The STEREO-HBM study is a multicenter 2-step nonrandomised phase II trial where 46 patients are planned to be enrolled (Fig. 1). The study is based on both tolerance and clinical efficacy as proposed by Bryant and Day's which allows simultaneous evaluation of clinical response and toxicity [13].

\section{Study sites}

The list of study sites is available on https://clinicaltrials. gov/ct2/show/NCT03696680.

\section{Study treatment}

Each targeted brain metastasis (hemorrhagic or not) will be treated at the dose of $30 \mathrm{~Gy}$ in 3 fractions at $10 \mathrm{~Gy} /$ fraction every 2 days $[19,20]$. All target lesions (maximum 3 brain metastases plus one tumor bed) will be treated as much as possible over 1 week. However, cerebral irradiation of all the lesions may be spread over 710 calendar days. The irradiation facility could be LINAC (Truebeam STX ${ }^{\circ}$, Versa $\mathrm{HD}^{\circ}$, Novalis ${ }^{\oplus} . .$. ) or robotic radiosurgery system (Cyberknife ${ }^{\circ}$.

Table 1 Study eligibility criteria

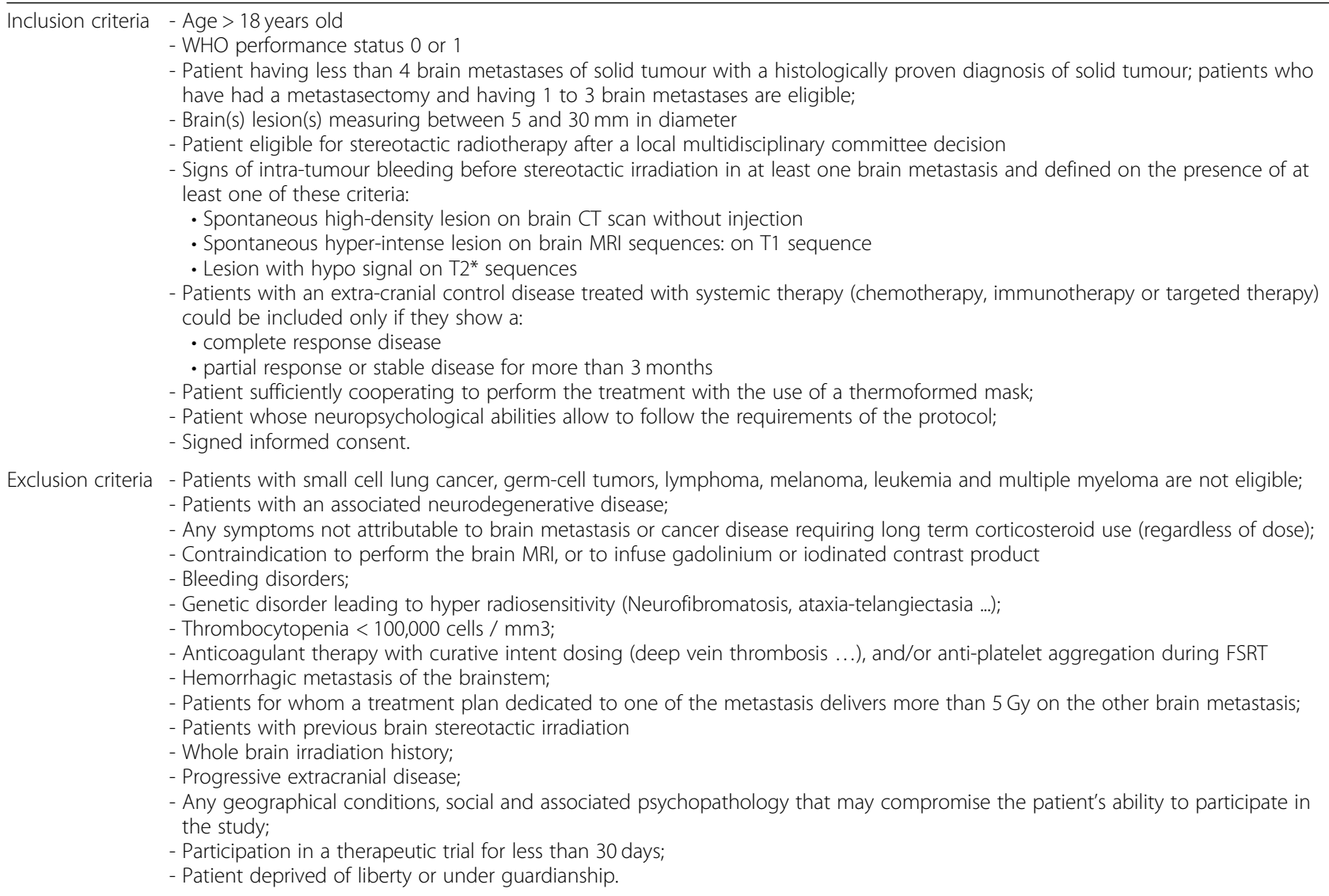




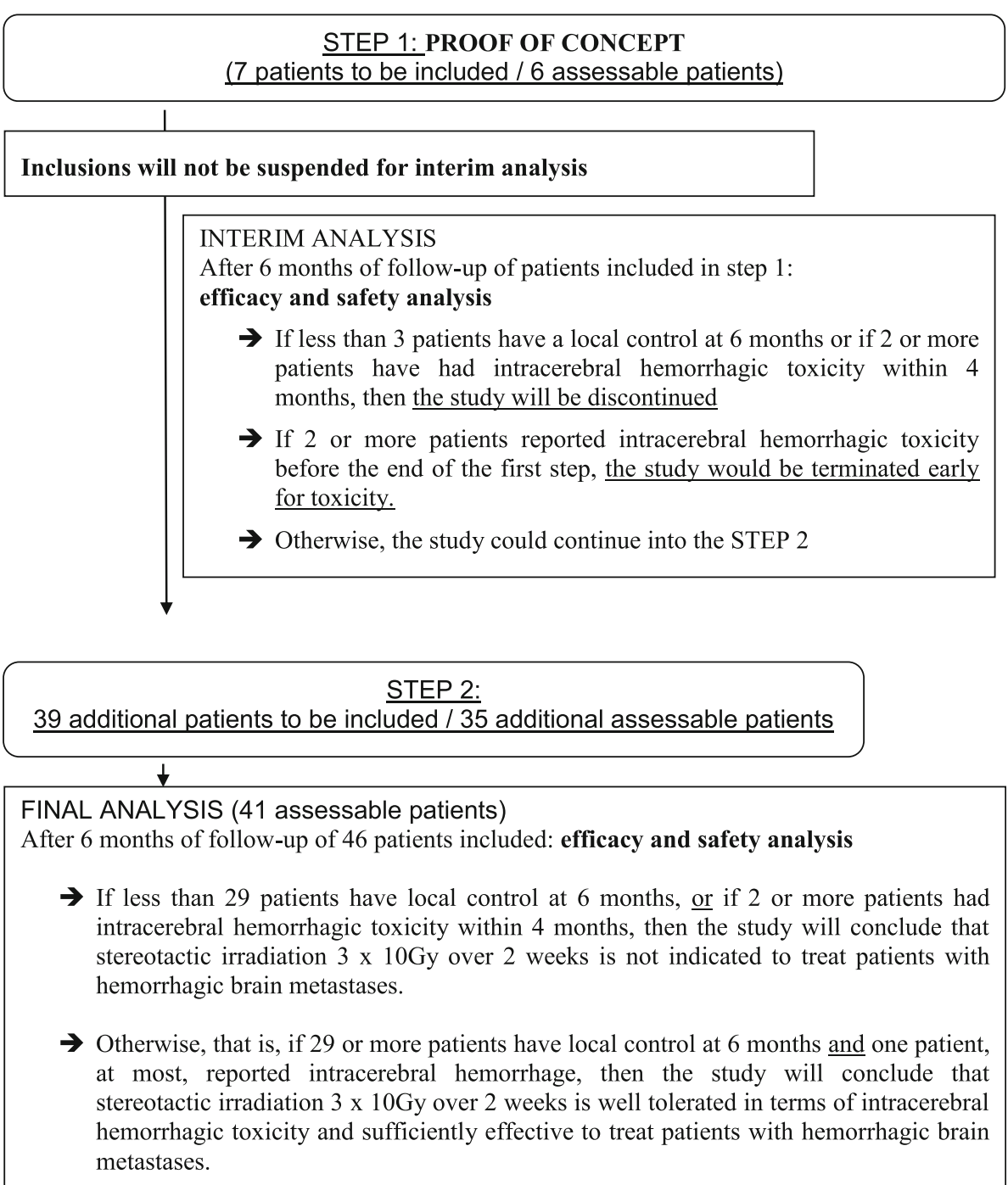

Fig. 1 Methodology design of the STEREO-HBM study

A minimum of $95 \%$ of the target volume (PTV) should receive at least $95 \%$ of the total prescribed dose of $30 \mathrm{~Gy}$ (V95 > 28.5Gy).

The target volumes will be defined as [21, 22]:

- GTV (Gross tumor volume): Gadolinium enhanced volume or surgical tumor bed

- $\mathrm{CTV}$ (clinical target volume $)=[\mathrm{GTV}+1 \mathrm{~mm}]$

- $\mathrm{SM}$ (set-up margins) $=1-2 \mathrm{~mm}$ according to the technique or irradiation system used

- $\mathrm{PTV}$ (planning target volumes $)=\mathrm{CTV}+\mathrm{SM}$

Organ at risk will be delineated according to investigator habits (Optic chiasm, Optic nerves, Brainstem, Cochlea, Spinal Cord, Eyes). The prescription isodose percentage should be higher than $70 \%$.

\section{Study procedures}

The trial schema is illustrated in Fig. 2. The overview of study assessments and procedures are detailed in Table 2.

\section{Brain tumor evaluation}

Brain tumoral evaluation will be in line with international guidelines [23]. It will be based on a brain MRI performed at baseline (before FSRT), at 1 week, 4 weeks, 8 weeks after the end of FSRT and thereafter at 4 months, 6 months and every 3 months post-FSRT in the absence of tumoral progression.

Each brain MRI will include the following sequences [19, 24, 25]: T1, T2, T2*, T1 with gadolinium and T2 FLAIR, and, if possible, MRI SWI (susceptibilityweighted imaging). 


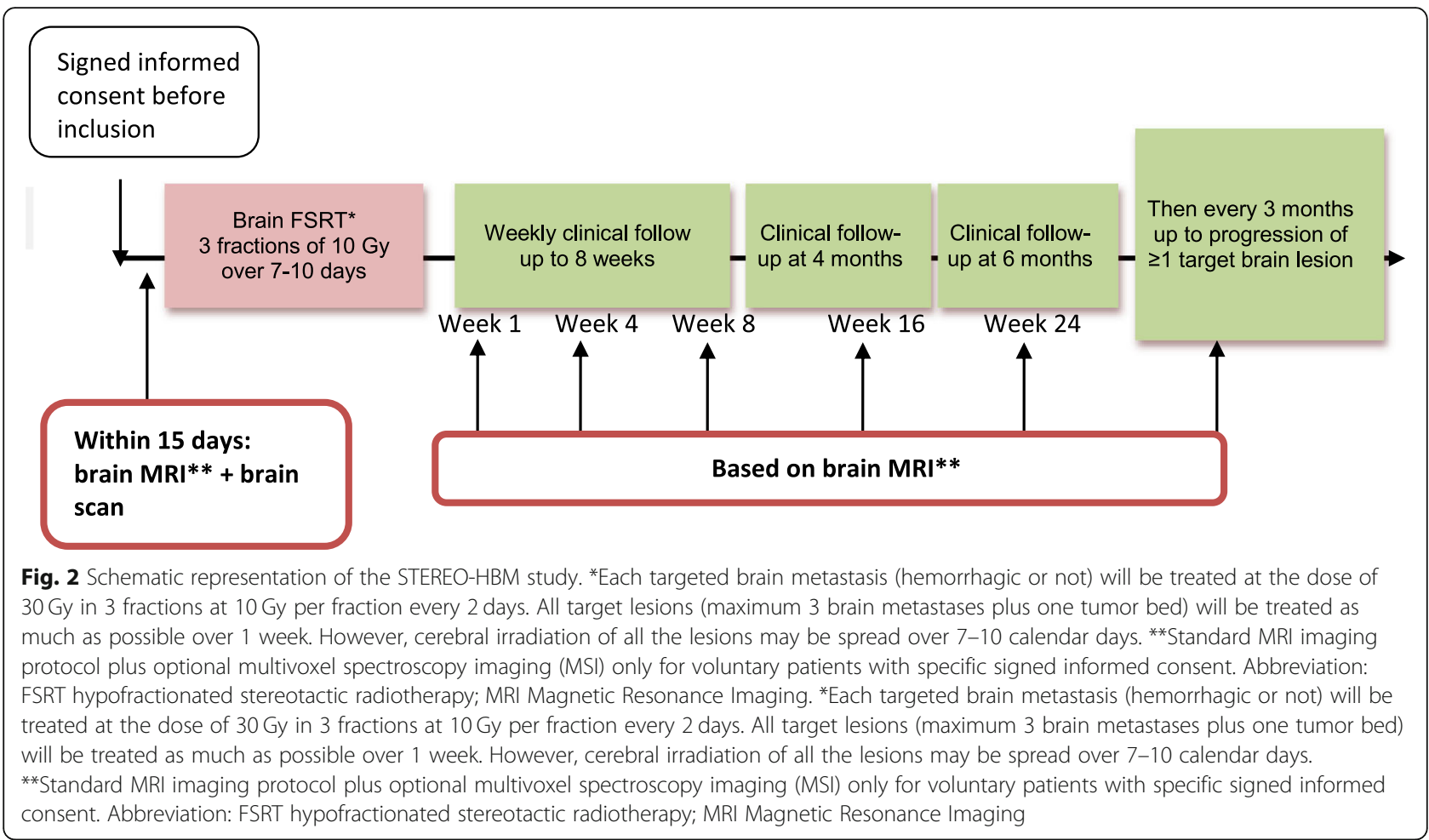

Disease assessment evaluation will be determined locally according to RECIST version 1.1 criteria.

\section{Multi-modality MRI ancillary study}

In addition to the standard MRI imaging protocol, each MRI imaging evaluation will include an optional multivoxel spectroscopy imaging (MSI) that will be performed only for voluntary patients with specific signed informed consent.

Perfusion and diffusion sequences will be added [26-28]. Evaluations may be helpful to explore the biochemistry of the tumor. Indeed, it appears important to be able to differentiate a tumor relapse from a therapeutic effect (radionecrosis) in the setting of this FSRT.

\section{Quality of life assessment}

Each patient will be asked to fil in standardized and validated self-administered questionnaires (EORTC QLQC30 and its specific brain cancer module BN-20) to assess health-related quality of life (QoL). QoL will be assessed at baseline, 4 weeks after the end of FSRT, thereafter 4 months, 6 months and every 3 months if no disease progression has occurred.

\section{Concomitant treatments}

Authorized concomitant treatments include bisphosphonates and corticotherapy, prescribed at the discretion of the investigator, according to local practices.

The following treatments are prohibited:
- Systemic anticancer drugs (including chemotherapy, hormonotherapy, anti-angiogenics) have to be suspended at least 7 days prior to FSRT initiation and may be reintroduced 7 days after the last fraction.

- Anticoagulant drugs taken in a curative intent and platelet anti-aggregants have to be suspended at least 5 days prior to FSRT initiation and may be reintroduced 2 months after the end of FSRT

\section{Statistical design overview}

The study will be conducted in 2 steps (a 'proof of concept' step followed by a 'validation' step) with a twostage phase 2 design proposed by Bryant and Day [13], combining both safety and efficacy as primary endpoint (Fig. 1).

We posited the following assumptions:

- $\pi_{\mathrm{T} 0} \geq 0.15$ and $\pi_{\mathrm{T} 1} \leq 0.05$, the unacceptable and expected rate of hemorrhagic complications occurring within 4 months after the end of FSRT, respectively

- $\pi_{\mathrm{R} 0} \leq 60 \%$ and $\pi_{\mathrm{R} 1} \geq 80 \%$, the unacceptable and expected local control rate of irradiated target lesions at 6 months, respectively.

With an alpha risk of $10 \%$ for both the efficacy and the toxicity, and a power of $90 \%$, a total of 41 assessable patients are required. 


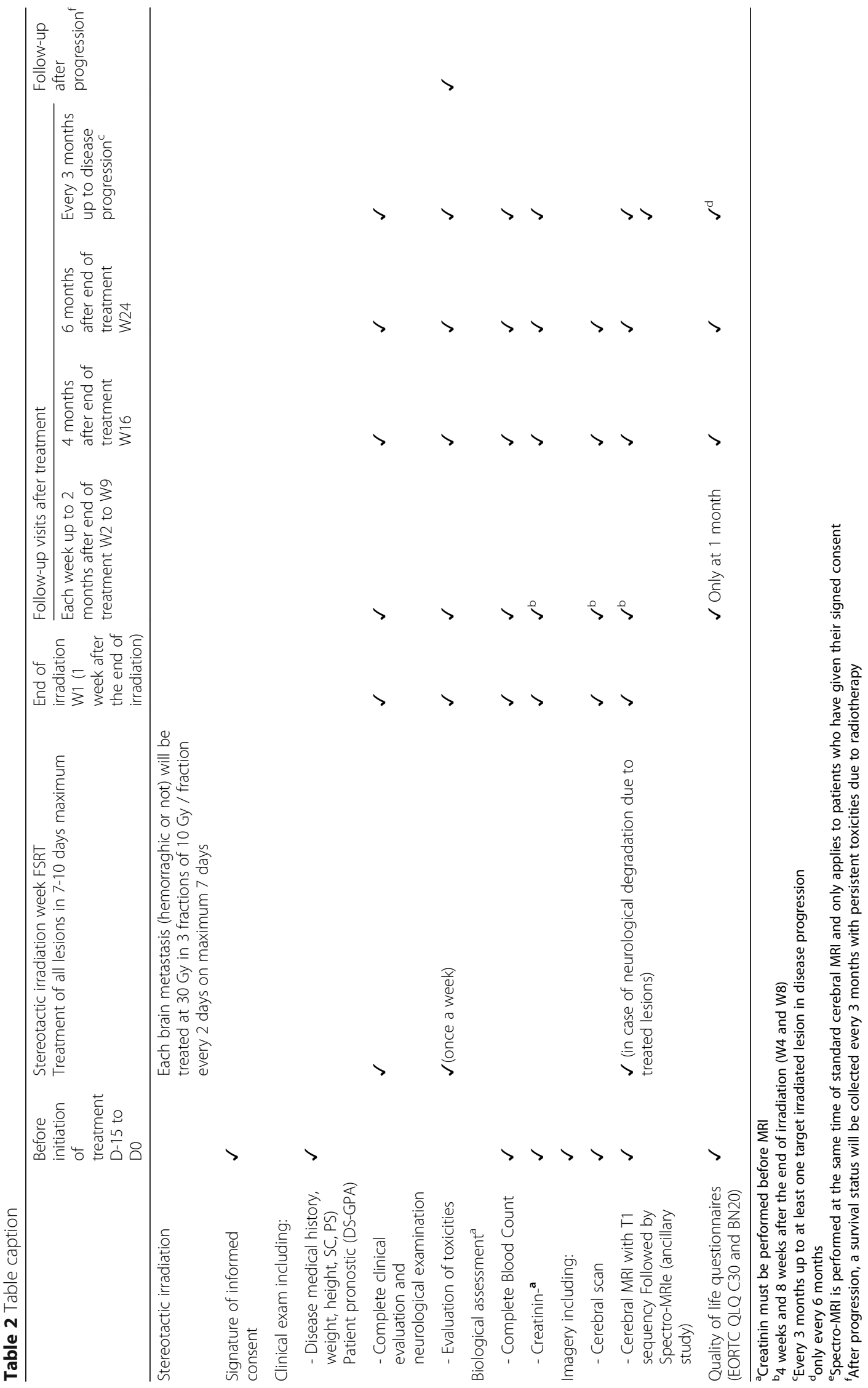


The continuation of the study will depend on the results of the interim analysis.

Interim analysis will be performed after the first step: 6 assessable patients will be analyzed. Inclusions will not be suspended during the interim analysis. If less than 3 patients are locally controlled at 6 months or if 2 or more patients have presented an intracerebral hemorrhagic toxicity within 4 months, then the study will be discontinued for futility. If 2 or more patients reported intracerebral hemorrhagic toxicity before the end of the first step, the study would be terminated early for excess of toxicity. Otherwise, the study could continue into the second step: 35 additional assessable patients will be needed.

Final analysis will be performed after the second step. After a 6-month follow-up of the 41 assessable patients, if less than 29 patients are locally controlled at 6 months, or if 2 or more patients had intracerebral hemorrhagic toxicity within the 4 months following FSRT, then the study will conclude that FSRT ( $3 \times 10$ Gy over 1 week) is not indicated to treat patients with hemorrhagic brain metastases. Otherwise, that is, if 29 or more patients are locally controlled at 6 months and if 1 patient, at most, reported intracerebral hemorrhage within 4 months post-FSRT, then the study will conclude that FSRT is effective, well tolerated and does not increase intracerebral hemorrhagic toxicity in patients with bleeding brain metastases.

Considering a drop-out rate of $10 \%$ (lost to follow-up, protocol deviation, etc.), 7 and 39 patients will be enrolled in the first and second step, respectively, for a total of 46 patients.

\section{Data management}

A Web Based Data Capture (WBDC) system will be used for data collection and query handling. The investigator will ensure that data are recorded on the eCRFs as specified in the study protocol and in accordance with the instructions provided.

The investigator ensures the accuracy, completeness, and timeliness of the data recorded and of the provision of answers to data queries according to the Clinical Study Agreement. The investigator will sign the completed eCRFs. A copy of the completed eCRFs will be archived at the study site.

\section{Data monitoring committee}

An Independent Data Monitoring Committee (IDMC) will be set-up to ensure the protection of patients, the ethical conduct of the study, to evaluate the benefit/risk ratio of the study, and to insure an independent review of the scientific outcomes during and at completion of the study. The IDMC exercises a consultative role for the promoter who takes the final decision for implementing the recommendations proposed by the IDMC.
The committee will include a radiotherapist, an oncologist, a statistician and a pharmacologist.

\section{Withdrawal from study}

Reasons for why a patient may discontinue participating to the study include:

- Patient request (withdrawal of consent for further treatment)

- Intolerable toxicity

- Concomitant disease or other reason requiring the discontinuation of treatment

- Patient lost to follow-up

- Investigator's request (with detailed documentation of reasoning)

\section{Discussion}

The scientific data studying the relationship between hypofractionated stereotactic radiotherapy (FSRT) or radiosurgery (SRS) for the management of hemorrhagic brain metastases, and the risk of intra-tumor and/or cerebral hemorrhage at the end of treatment are very insufficient, or contradictory.

In this context, we aim at assessing the interest of FSRT by proposing the first prospective phase 2 trial focusing on both safety and efficacy of this strategy for patients with bleeding brain metastasis.

In addition, intra-tumoral hemorrhagic events before and after treatment will be precisely documented, using multi-modal MRI. Patients' health-related quality of life before and after treatment will be also assessed, using standardized validated self-administered questionnaires.

This project comes within a large scientific program of our Institution that aims at assessing various treatment approaches in primary and secondary brain tumours [29].

In the future, we hope the results of our prospective trial will reinforce that patients with hemorrhagic brain metastases could benefit from adapted and innovated treatment like FSRT, for optimal and safe management allowing maintaining quality of life.

\section{Abbreviations \\ ANOCEF: Association des Neuro-Oncologues d'expression française/Associ- ation of the neuro-oncologists of French expression; CTV: Clinical target volume; EORTC: European Organisation for Research and Treatment of Cancer; FSRT: Stereotactic radiotherapy; GTV: Gross tumor volume; HAS: French High Authority of Health; IDMC: Independent Data Monitoring Committee; MRI: Magnetic Resonance Imaging; MSI: Multivoxel spectroscopy imaging; PTV: Planning target volumes; QoL: Quality of Life; SM: Set-up margins; SPIRIT: Standard protocol items, namely recommendations for interventional trials; SRS: Radiosurgery; WBDC: Web Based Data Capture; WBRT: Whole Brain radiotherapy; WHO: World Health Organization}

\section{Acknowledgements}

We are grateful to the members of the Independent Data Monitoring Committee. We acknowledge the ANOCEF (Association des NeuroOncologues d'expression française/Association of the neuro-oncologists of French expression) for its support in scientific collaboration. We thank the 
Data Processing Centre (DPC) of the North West Canceropole (Centre de Traitement des Données du Cancéropôle Nord-Ouest) in charge of data management. The investigators are also thanked, namely loana Hrab, Philippe Royer, Guillaume Vogin, Valérie Bernier, Myriam Khadige.

\section{Authors' contributions}

$A L, D S, P L$, and $B C$ wrote the manuscript and devised the study concept and design. JLe were responsible for overseeing the statistical section. JG, PEB, WK, and PR have been involved in drafting the manuscript or revising it critically for important intellectual content. DS and $B C$ supervised the entire work. All authors (PL, WK, AL, JG, JLe, JLa, PEB, IH, PR, BC, DS) have given final approval of the version to be published. Each author has participated sufficiently in the work to take public responsibility for appropriate portions of the content.

\section{Funding}

This trial (NCT03696680) is granted by the French Health Ministry through North West interregional hospital clinical research program (PHRCl-17-087). In the context of this major external funding, the study protocol has undergone peer-review by the funding body.

The funding agency was not involved in the design and conduct of the study, nor in the collection, management, analysis, and interpretation of the data. It was not involved in the writing of the manuscript.

\section{Availability of data and materials}

Not applicable.

\section{Ethics approval and consent to participate}

This study has received ethical approval from the Comité de Protection des Personnes Sud-est 2 in September 2018 ( N ID-RCB: 2018-A00926-49) and from National Agency for Medical and Health products Safety in July 2019. All patients will give their written informed consent before any study-related assessment start.

\section{Consent for publication}

Not applicable.

\section{Competing interests}

The authors declare that they have no competing interests.

\section{Author details}

${ }^{1}$ Radiation Oncology Department, Centre François Baclesse, F-14000 Caen, France. ${ }^{2}$ Normandy University, F-14000 Caen, France. ${ }^{3}$ Clinical Research Department, Centre François Baclesse, F-14000 Caen, France. ${ }^{4}$ Radiology Department, Centre François Baclesse, F-14000 Caen, France. ${ }^{5}$ Medical Oncology Department, Centre François Baclesse, F-14000 Caen, France. ${ }^{6}$ Radiation Oncology Department, Institut de Cancérologie de Lorraine, F-54000 Vandœuvre-lès-Nancy, France. ${ }^{7}$ Radiation Oncology Department, Centre François Baclesse, 3 Avenue du Général Harris, F-14076 Caen Cedex 05, France.

Received: 6 December 2019 Accepted: 21 January 2020

Published online: 22 February 2020

\section{References}

1. Soffietti R, Rudā R, Mutani R. Management of brain metastases. J Neurol. 2002;249:1357-69.

2. Madajewicz S, Karakousis C, West CR, Caracandas J, Avellanosa AM. Malignant melanoma brain metastases. Review of Roswell Park Memorial Institute experience. Cancer. 1984;53:2550-2.

3. Posner JB. Diagnosis and treatment of metastases to the brain. Clin Bull. 1974:4:47-57.

4. Borgelt B, Gelber R, Kramer S, Brady LW, Chang CH, Davis LW, et al. The palliation of brain metastases: final results of the first two studies by the radiation therapy oncology group. Int J Radiat Oncol Biol Phys. 1980;6:1-9.

5. Sampson JH, Carter JH, Friedman AH, Seigler HF. Demographics, prognosis, and therapy in 702 patients with brain metastases from malignant melanoma. J Neurosurg. 1998:88:11-20.

6. Wakai S, Yamakawa K, Manaka S, Takakura K. Spontaneous intracranial hemorrhage caused by brain tumor: its incidence and clinical significance. Neurosurgery. 1982;10:437-44.
7. Kondziolka D, Bernstein M, Resch L, Tator CH, Fleming JF, Vanderlinden RG, et al. Significance of hemorrhage into brain tumors: clinicopathological study. J Neurosurg. 1987;67:852-7.

8. Aoyama H, Shirato H, Tago M, Nakagawa K, Toyoda T, Hatano K, et al. Stereotactic radiosurgery plus whole-brain radiation therapy vs stereotactic radiosurgery alone for treatment of brain metastases: a randomized controlled trial. JAMA. 2006;295:2483-91.

9. Meyners T, Heisterkamp C, Kueter J-D, Veninga T, Stalpers LJA, Schild SE et al. Prognostic factors for outcomes after whole-brain irradiation of brain metastases from relatively radioresistant tumors: a retrospective analysis. BMC Cancer. 2010;10:582.

10. Suzuki H. Spontaneous haemorrhage into metastatic brain tumours after stereotactic radiosurgery using a linear accelerator. J Neurol Neurosurg Psychiatry. 2003;74:908-12.

11. Redmond AJ, Diluna ML, Hebert R, Moliterno JA, Desai R, Knisely JPS, et al. Gamma knife surgery for the treatment of melanoma metastases: the effect of intratumoral hemorrhage on survival. J Neurosurg. 2008;109(Suppl):99-105.

12. Mathieu D, Kondziolka D, Cooper PB, Flickinger JC, Niranjan A, Agarwala S, Kirkwood J, Lunsford LD. Gamma knife radiosurgery for malignant melanoma brain metastases. Clin Neurosurg. 2007;54:241-7

13. Bryant J, Day R. Incorporating toxicity considerations into the design of twostage phase II clinical trials. Biometrics. 1995;51(4):1372-83.

14. Chang EL, Selek U, Hassenbusch SJ 3rd, Maor MH, Allen PK, Mahajan A, Sawaya R, Woo SY. Outcome variation among "radioresistant" brain metastases treated with stereotactic radiosurgery. Neurosurgery. 2005;56(5):936-45.

15. Mathieu D, Kondziolka D, Cooper PB, Flickinger JC, Niranjan A, Agarwala S, Kirkwood J, Lunsford LD. Gamma knife radiosurgery in the management of malignant melanoma brain metastases. Neurosurgery. 2007;60(3):471-81; discussion 481-2.

16. Sahgal A, Larson D, Knisely J. Stereotactic radiosurgery alone for brain metastases. Lancet Oncol. 2015;16(3):249-50. https://doi.org/10.1016/S14702045(14)71106-4.

17. Yamamoto M, Serizawa $T$, Shuto $T$, et al. Stereotactic radiosurgery for patients with multiple brain metastases (JLGK0901): a multi-institutional prospective observational study. Lancet Oncol. 2014;15(4):387-95.

18. Zhang J, Zhang ZB, Gao H, Zhang D, Wang WL. Haemorrhage detection in brain metastases of lung cancer patients using magnetic resonance imaging. Int Med Res. 2009;37(6):1842-50.

19. Wiggenraad R, Bos P, Verbeek-de Kanter A, Lycklama À, Nijeholt G, van Santvoort J, Taphoorn M, Struikmans H. Pseudo-progression after stereotactic radiotherapy of brain metastases: lesion analysis using MRI cineloops. J Neuro-Oncol. 2014;119(2):437-43.

20. Shaw E, Scott C, Souhami L, Dinapoli R, Kline R, Loeffler J, Farnan N. Single dose radiosurgical treatment of recurrent previously irradiated primary brain tumors and brain metastases: final report of RTOG protocol 90-05. Int J Radiat Oncol Biol Phys. 2000;47(2):291-8.

21. Noël G, Simon JM, Valery CA, Cornu P, Boisserie G, Hasboun D, Ledu D, Tep B, Delattre JY, Marsault C, Baillet F, Mazeron JJ. Radiosurgery for brain metastasis: impact of CTV on local control. Radiother Oncol. 2003;68(1):15-21.

22. Chang SD, Main W, Martin DP, Gibbs IC, Heilbrun MP. An analysis of the accuracy of the CyberKnife: a robotic frameless stereotactic radiosurgical system. Neurosurgery. 2003;52(1):140-6 discussion 146-7.

23. Ellingson BM, Bendszus M, Boxerman J, Barboriak D, Erickson BJ, Smits M, Nelson SJ, Gerstner E, Alexander B, Goldmacher G, Wick W, Vogelbaum M, Weller M, Galanis E, Kalpathy-Cramer J, Shankar L, Jacobs P, Pope WB, Yang D, Chung C, Knopp MV, Cha S, van den Bent MJ, Chang S, Yung WK, Cloughesy TF, Wen PY, Gilbert MR. Consensus recommendations for a standardized brain tumor imaging protocol in clinical trials. NeuroOncology. 2015;17(9):1188-98.

24. Doré M, Lefebvre L, Delpon G, Thillays F. Brain radiation necrosis after stereotactic radiotherapy of the resection cavity for intracranial metastases: analysis of the literature from four cases. Cancer Radiother. 2015;19(2):111-9.

25. Patsouris A, Augereau P, Tanguy JY, Morel O, Menei P, Rousseau A, Paumier A. Differential diagnosis of local tumor recurrence or radionecrosis after stereotactic radiosurgery for treatment of brain metastasis. Cancer Radiother. 2014;18(2):142-6.

26. Almeida-Freitas DB, Pinho MC, Otaduy MCG, Braga HF, Meira-Freitas D, da Costa Leite C. Assessment of irradiated brain metastases using dynamic contrast-enhanced magnetic resonance imaging. Neuroradiology. 2014; 56(6):437-43. 
27. Koh MJ, Kim HS, Choi CG, Kim SJ. Which is the best advanced MR imaging protocol for predicting recurrent metastatic brain tumor following gammaknife radiosurgery: focused on perfusion method. Neuroradiology. 2015; 57(4):367-76.

28. Jakubovic R, Sahgal A, Soliman H, Milwid R, Zhang L, Eilaghi A, Aviv RI. Magnetic resonance imaging-based tumour perfusion parameters are biomarkers predicting response after radiation to brain metastases. Clin Oncol (R Coll Radiol). 2014;26(11):704-12.

29. Lesueur P, Lequesne J, Grellard JM, Dugué A, Coquan E, Brachet PE, Geffrelot J, Kao W, Emery E, Berro DH, Castera L, Goardon N, Lacroix J, Lange M, Capel A, Leconte A, Andre B, Léger A, Lelaidier A, Clarisse B, Stefan D. Phase I/lla study of concomitant radiotherapy with olaparib and temozolomide in unresectable or partially resectable glioblastoma: OLATMZ-RTE-01 trial protocol. BMC Cancer. 2019;19(1):198.

\section{Publisher's Note}

Springer Nature remains neutral with regard to jurisdictional claims in published maps and institutional affiliations.

Ready to submit your research? Choose BMC and benefit from:

- fast, convenient online submission

- thorough peer review by experienced researchers in your field

- rapid publication on acceptance

- support for research data, including large and complex data types

- gold Open Access which fosters wider collaboration and increased citations

- maximum visibility for your research: over $100 \mathrm{M}$ website views per year

At $B M C$, research is always in progress.

Learn more biomedcentral.com/submissions 